\title{
Statistical Reconstruction Methods in PET: Resolution Limit, Noise, Edge Artifacts and considerations for the design of better scanners
}

\author{
J. L. Herraiz ${ }^{1}$, S. España ${ }^{1}$, J. M. Udías ${ }^{1}$, J. J. Vaquero ${ }^{2}$, M. Desco ${ }^{2}$ \\ ${ }^{1}$ Dpto. Física Atómica, Molecular y Nuclear, Complutense University, Madrid, Spain \\ ${ }^{2}$ Unidad de Medicina y Cirugía Experimental, Hospital GU “Gregorio Marañón”, Madrid, Spain
}

\begin{abstract}
Small animal positron emission tomography (PET) scanners are being increasingly used as a basic measurement tool in modern biomedical research. The new designs and technologies of these scanners and the modern reconstruction methods have allowed to reach high spatial resolution and sensitivity. Despite their successes, some important issues remain to be addressed in high resolution PET imaging. First, iterative reconstruction methods like maximum likelihood-expectation maximization (MLEM) are known to recover resolution, but also to create noisy images and edge artifacts if some kind of regularization is not imposed. Second, the limit of resolution achievable by iterative methods on high resolution scanners is not quantitatively understood. Third, the use of regularization methods like Sieves or maximum a posteriori (MAP) requires the determination of the optimal values of several adjustable parameter that may be object-dependent.
\end{abstract}

In this work we review these problems in high resolution PET and establish that the origin of them is more related with the physical effects involved in the emission and detection of the radiation during the acquisition than with the kind of iterative reconstruction method chosen. These physical effects (positron range, non-collinearity, scatter inside the object and inside the detector materials) cause that the tube of response (TOR) that connects the voxels with a line of response (LOR) is rather thick. This implies that the higher frequencies of the patient organ structures are not recorded by the scanner and therefore cannot be recovered during the reconstruction. As iterations grow, MLEM algorithms try to recover higher frequencies in the image. Once that a certain critic frequency is reached, this only maximizes high frequency noise.

Using frequency response analyses techniques, we determine the maximum achievable resolution, before edge artifacts spoil the quality of the image, for a particular scanner as a function of the thickness of the TOR, and independently of the reconstruction method employed. With the same techniques, we can deduce well defined stopping criteria for reconstructions methods. Also, criteria for the highest number of subsets which should be used and how the design of the scanners can be

Manuscript received November 11, 2005. J.L. Herraiz acknowledges support from UCM grant. e mail: joaquin@nuc1.fis.ucm.es. S. España acknowledges support from "Fundacion para la investigación biomédica del Hospital Gregorio Marañon” grant. e mail: samuel@nuc1.fis.ucm.es. J.J.Vaquero e mail: juanjo@dns.hggm.es M. Desco e mail: desco@mce hggm.es J.M. Udias e mail: jose@nuc2.fis.ucm.es. acknowledges support from MEC project (BFM2003 04147 C02 01). Acknowledges for "Ultra micro resolution phantom" data acquisition to Drs Yuchuan Wang and Jurgen Seidel, Department of Radiology, Johns Hopkins Medical Institution. optimized when statistical reconstruction methods are employed, is established.

Index Terms Image reconstruction, Positron emission tomography, Resolution limit, Noise, Iterative Methods, MLEM, OSEM.

\section{INTRODUCTION}

$\mathrm{D}$ URING the last years, dedicated small PET animal scanners have become one of the main tools in biomedical research. In this kind of studies, spatial resolutions on the order of $1 \mathrm{~mm}$ and high sensitivity are required. New technologies and reconstruction methods have been developed for reaching these goal. Iterative statistical reconstructions methods like ML-EM [1], [2] have shown superior image quality to conventional analytic reconstruction techniques, but despite their widespread and now conventional use, there are some important properties and limitations of these methods that have not been properly outlined so far:

I. The reconstruction problem is said to be ill-posed, because the noise in the reconstructed images increases as the number of iterations proceeds. There exists a paradox in the ML-EM method, because maximizing the log-likelyhood until convergence is reached creates noisy images [3]. Stopping the iterations before convergence makes the reconstructed images to be biased towards the initial starting image and furthermore optimal resolution can not be achieved. The origin of this problem is not quite explained in the literature.

II. They have been proposed many different methods for avoiding noise as well as edge artefacts, like the method of Sieves [4], replacing the maximum-likelihood criterion with a penalized-likelihood (or MAP) objective function [5], or filtering the image during the iterations or post-smoothing it [6]. All these methods have free parameters that need to be determined. The optimal value of the parameters is in general object-dependent and this reduces the usefulness of these methods in clinical studies.

III. With iterative methods, the choice of the number of voxels of the reconstructed images seems to be quite arbitrary, but in fact, there is an optimal resolution for each kind of scanner.

IV. Finding the optimal number of subsets in the accelerated version of the algorithm and the stopping criteria for the iterative methods is still an open issue. 
V. More often, scanners have been designed with analytic reconstruction methods in mind. This doesn't imply that such designs are the best ones for statistical reconstruction of PET images. Now that statistical methods are being commonly used, new scanners, specifically designed to take advantage of these reconstruction methods must be thought of.

The majority of available PET scanners are configured as small individual detector units in an array of scintillator crystals. Bearing in mind all the physical effects (positron range,non-collinearity, scatter of the gamma rays inside the object and inside the crystals) and electronic noise that are involved in the data acquisition of a PET scanner, the volume of space from which an emitted positron can produce coincidence counts in a pair of crystals is rather large, measured in crystal units. This volume is in general distributed on a tube of response (TOR) along the line of response (LOR).

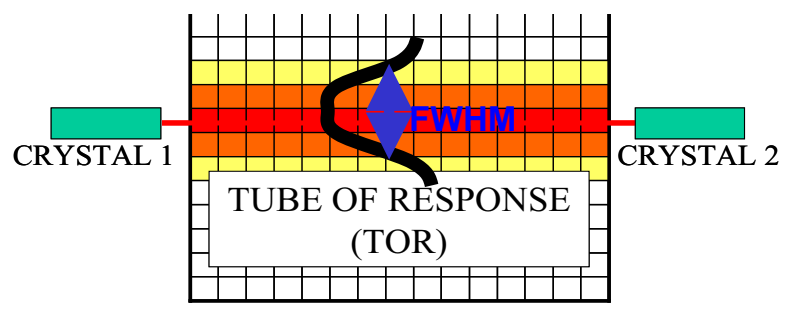

Fig. 1. Squematic drawing of a Tube of Response (TOR)

In small PET animal scanners, the size of the TORs determines their main properties, like maximum resolution or the signal to noise ratio achievable, to a much larger extent than the number of LORs or the distance among them.

\section{FREQUENCY ANALYSIS OF PET DATA}

The sizeable width of the TOR causes the scanner to be blind to high frequency components of the object [3], even if the sampling is increased by using a very large number of crystals. The FWHM of the TOR can be compared to the Modulation Transfer Function [7] employed for characterizing optical systems such as video cameras or film scanners.

One can define the maximum system frequency (MSF) as the highest frequency of the object that the scanner can record for the ideal case of a noise-free acquisition. When noise is present, the maximum effective system frequency (MESF) can also be defined, as the highest frequency of the object that can be recorded above the noise. Analytical estimates of the MSF can be obtained to determine the maximum resolution achievable by any means, from a particular acquisition.

The average of the data in a LOR Y with sinogram coordinates $(\rho, \theta)$ is:

$$
Y(\rho, \theta)=\sum_{V \in T O R(\rho, \theta)} C(\rho, \theta ; V) \cdot X(V)
$$

$\mathrm{C}(\mathrm{r}, \mathrm{q} ; \mathrm{V})=$ System response matrix $(\mathrm{SRM})[$ In simple 2-D cases, the dependence on the distance from the line that connects two crystals can be assumed to be Gaussian]. $\mathrm{X}(\mathrm{V})=$ Reconstructed Image at voxel $\mathrm{V}$

For an ideal noise-free acquisition, if we assume that every
TORs has a transversal gaussian shape with the same FWHM, the MSF of the scanner is given by:

$$
\operatorname{MSF}\left(\mathrm{mm}^{-1}\right)=\frac{2 \cdot \sqrt{\operatorname{Ln} 2 \cdot \operatorname{Ln} 10}}{\pi \cdot F W H M(\mathrm{~mm})}
$$

PROOF: For the sake of simplicity only the 1-Dimension case is discussed here, naming $\rho$ the $\mathrm{x}$ coordinate. The SRM can then be assumed of the form: $C(\rho)=g(x)=\exp (-(x / a) 2)$ and its Fourier transform as $G(\omega)=\exp (-(a \omega / 2) 2)$. Using the relation $\omega=2 \pi f$, one has $G(f)=\exp (-(\pi \mathrm{af}) 2)$ (Fig. 2 in red). The Half Width Tenth Maximum of $G(f)$ is what we have been called MSF.

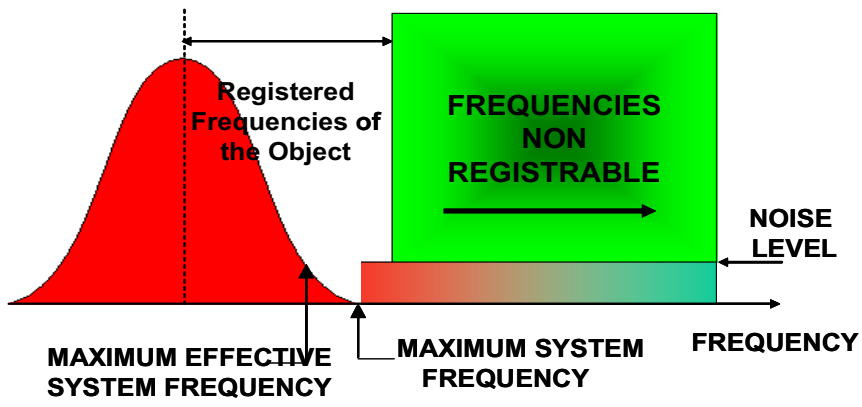

Fig. 2. Schematic drawing of the effect of the width of the TORs on the frequencies registered by a PET scanner.

In small animal PET scanners, the MSF usually lies far below the sampling frequency ( $\mathrm{F}_{\mathrm{NYQUIST}}$ ) so that, contrarily to analytical methods, aliasing is not the main limitation factor for resolution recovery in the case of iterative algorithms.

The best resolution achievable for a given MSF is:

$$
\Delta x(m m)=\frac{1}{2 \cdot M S F\left(m^{-1}\right)} \simeq 0.62 \cdot F W H M(m m)(3)
$$

For example, in an ideal scanner with TORs of $1.5 \mathrm{~mm}$ (FWHM) the best achievable resolution, by ANY algorithm would be $0.93 \mathrm{~mm}$. This also implies that the reconstructed images should have voxels of $0.46 \mathrm{~mm}$ in order to avoid grid aliasing.
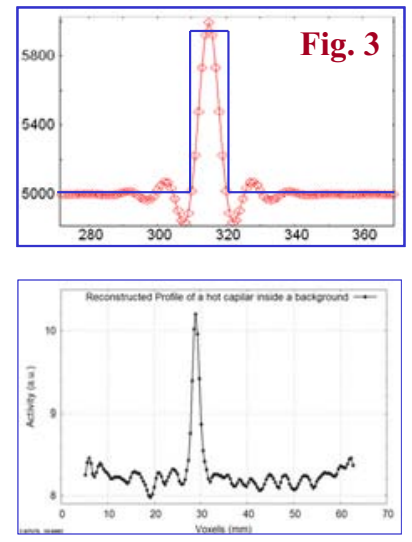

Fig. 3. Profile of reconstructed images of a capilar with background activity from simulated data (top) and a real acquisition (bottom).

The loss of higher frequencies of the object due to physical effects can be easily observed. Using a phantom with sharp edges, the reconstructed image will exhibit the 'Gibbs' 
phenomena, or ringing artifacts. Fig. 3 shows the reconstruction from a simulation (left) and a real acquisition (right) of a capilar with background activity. It corresponds to the response of a low-pass filtered delta function rather than to a gaussian.

\section{SIMULATIONS}

Simulations have been performed to test the predictions of the frequency analysis for iterative reconstructions. Fig. 4 presents a 2-Dimensional cosine phantom (above) which contains only one single frequency component, beyond the MSF limit. It can be seen that the system is blind to this frequency and completely fails to reconstruct the phantom (below).

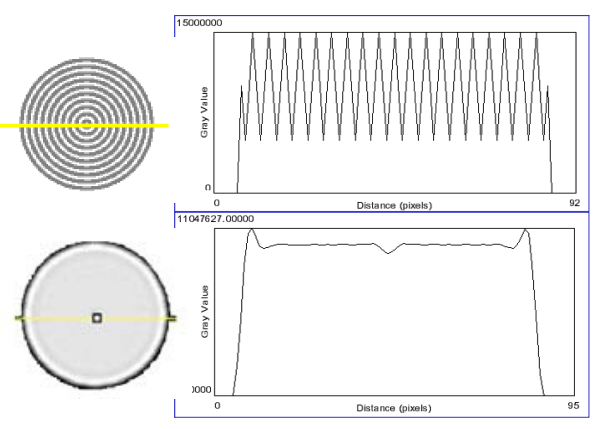

Fig.4. Cosine Phantom. Proyected phantom (top) and reconstructed image (bottom). The cosine frequency can't be recovered as it was not registered by the scanner.

\section{ORIGIN OF NOISE IN EM-ML RECONSTRUCTIONS}

EM-ML is said to be ill-posed because by maximizing the Log-likelihood (5.f), the noise in the reconstructed image increases as the number of iterations progress (5.i). This problem is studied here with the help of simulated acquisitions, including Poisson noise, of a cold-rod phantom (Fig. 5). The images reconstructed after 20 iterations (Iter.20) exhibit the lowest noise level and after 200 iterations (Iter.200), the best contrast recovery.

As the iteration number progress, statistical methods like ML-EM try to recover higher and higher frequencies (see the Power Spectrum 5.g, 5.h), even after the MSF is reached. Recovery of frequencies larger than the MSF can only be achieved by amplifying the higher frequency components of the acquisition (noise), because these components of the object will hardly be recorded by the scanner, as they are filtered by the convolution with the gaussian shape of the TOR during the forward projection. Only the noise contribution to the image increases.

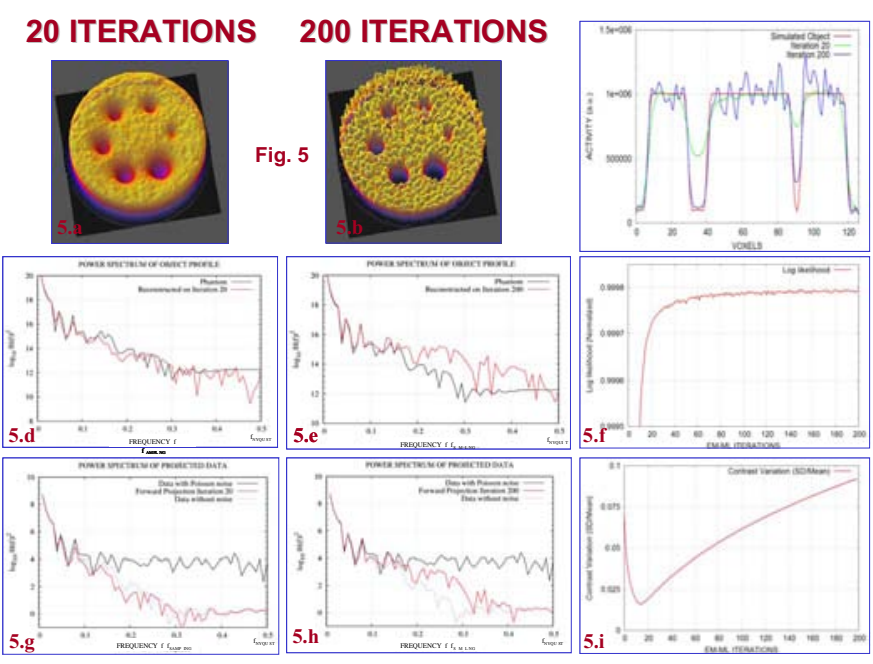

Fig. 5. Results from a cold rod phantom simulation. Reconstructed images and its profile (top), Power Spectrum of these images (middle) and Power Spectrum of the projected data and the Forward Projection (bottom).

\section{SAMPLING CONSIDERATIONS}

As sampling is not the main limitation in iterative reconstruction methods, there exists a need for a quantitative determination of the minimal number of LORs needed for an optimal reconstruction.

Some simulations have been done in order to estimate the minimum number of LORs required. First, an ideal noise-free case where the data was obtained forward-projecting the coldrod phantom with the SRM was considered. To analyze the effect of noise, the same study was applied to a case with Poisson noise added to data.

Performance was evaluated by means of two figures of merit: A) Cold Contrast Recovery (CCR) in a Region of Interest (ROI), in this case, the cold rods: $\mathrm{CCR}=$ (Reconstructed(Cold ROI)-Mean(Cold ROI)) / Mean(BG) B) Background Noise: Standard Deviation (SD) from the BG normalized to the BG mean.

In Fig. 6 we show the main results of this optimal sampling study. Higher sampling doesn't improve the quality of the image reconstructed from noiseless data and reducing the number of radial bins worsens the image more than reducing the number of projections. An optimal value for the number of radial bins and projection can be considered: $\mathrm{n}_{\mathrm{r}}=\mathrm{n}_{\mathrm{a}}=$ FOV $/$ Pixel Size.

As can be seen in Fig. 6, the conclusions about the optimal sampling obtained in an ideal case, can not be easily extrapolated to realistic data, as they will be noise-dependent. 


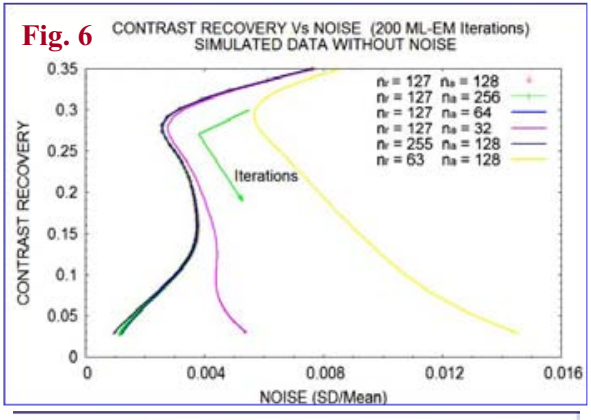

CONTRAST RECOVERY VS NOISE (200 ML-EM Iterations)

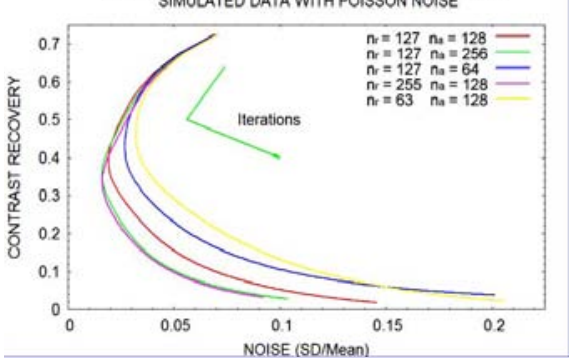

Fig. 6. Contrast recovery versus Noise for reconstructions of the above cold rods phantom with noiseless data (left) and data with Poisson noise (right). The number of projections $\left(n_{a}\right)$ and radial bins $\left(n_{r}\right)$ of a sinogram are varied. The number of emitted counts was the same in all the simulations.

\section{OTHER PARAMETERS OF THE RECONSTRUCTION}

Not only the optimal resolution can be determined by this analytical formulation. In this kind of scanners, resolution is more constrained by the form of the matrix of response of the system than by sampling. Filtered Back-Projection (FBP) method has better results when sampling is improved, using more LORs, as it avoids aliasing [8]. On the contrary, in MLEM, aliasing is not a main limitation, as the width of the TORs is a much more important factor. This is what allows reconstructing iteratively using $\mathrm{N}$ subsets of data. In each subset, the sampling is decreased by a factor $\mathrm{N}$, and the signal to noise level is decreased. The maximum number of Subsets that can be chosen without compromising the quality of the image can be determined using this kind of analysis.

From this framework, regulatization and stopping rule problems can also be dealt with. The knowledge of the higher recoverable frequency can also allow to create well defined regularization methods.

\section{REAL DATA}

Statistical algorithms can be considered as a method of deconvolution of projected data. The width of the TOR used for the reconstruction determines the maximum frequency that will be recovered. Wide TORs will yield poor resolution reconstructions, but with low noise levels. On the other hand, thin TORs (the usual choice when a simplified response of the system is employed) will force the reconstruction to try to recover higher frequencies, resulting in an increase of the noise level.

The effect of the width of the TOR used for the reconstruction was studied (see Figs. 7 and 8 ) for an acquisition obtained from a commercially available GE eXplore Vista scanner, at Johns Hopkins University. A Micro Ultra Resolution Phantom was acquired with 200 counts per LOR and coincidence events within a 400-650 keV energy window were accepted.

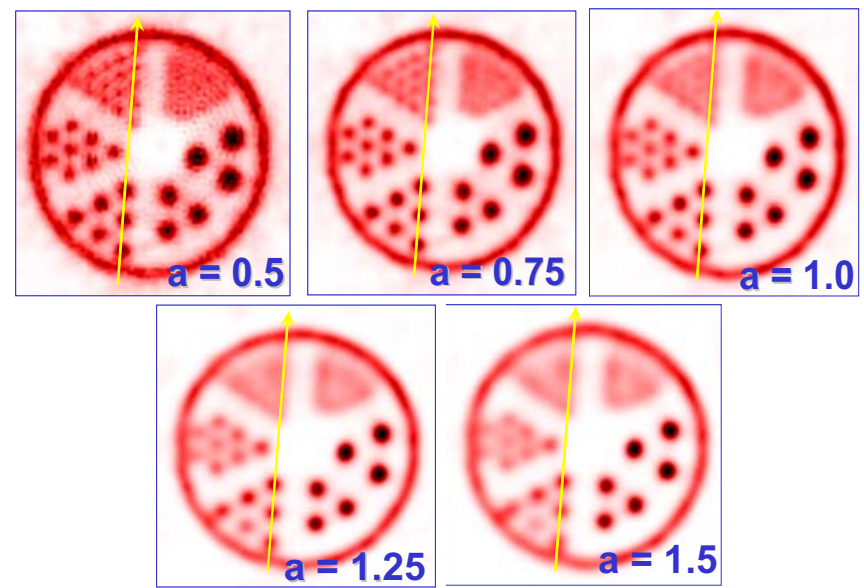

Fig. 7. Images of the Ultra Micro Resolution Phantom reconstructed with FIRST ${ }^{\circ}[9]$, using different width of the TORs. The diameters of the rods are $0.8,1.0,1.2,1.4,1.6$ and $1.8 \mathrm{~mm}$. Separation twice diameter. The results for one of the 61 slices through the axial FOV are shown.

The System Response Matrix employed for the reconstruction is obtained from a realistic MonteCarlo simulation which includes geometry, positron range, non collinearity and scatter in the detector crystals. Furthermore, reconstructions obtained with a modified SRM (scaling the FWHM of the TORs by a factor "a", corresponding a=1 to the realistic SRM) are also displayed.

With $\mathrm{a}=0.5$ or lower, i.e. using TORs much thinner than the realistic ones, aliasing artifacts appear. It is also worth to point out that $\mathrm{a}=0.75$ yields better image quality (measured both in $\mathrm{s} / \mathrm{n}$ ratio and resolution) than the realistic width $\mathrm{a}=$ 1.0 .

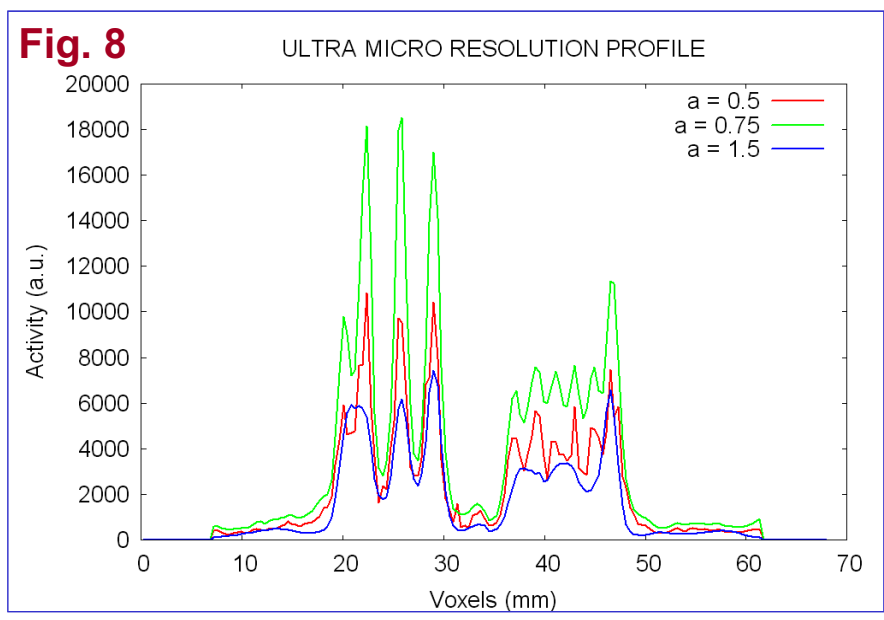

Fig. 8. Profiles of the reconstructed images of the Ultra Micro Resolution Phantom acquisition with different TOR widths. 
Fig. 9 presents the Power Spectrum of a projection for this phantom. Note that the only strength at high frequencies is due to the noise in the acquisition. Orange, Blue and Brown lines represent the limit of frequency recoverable with each SRM.

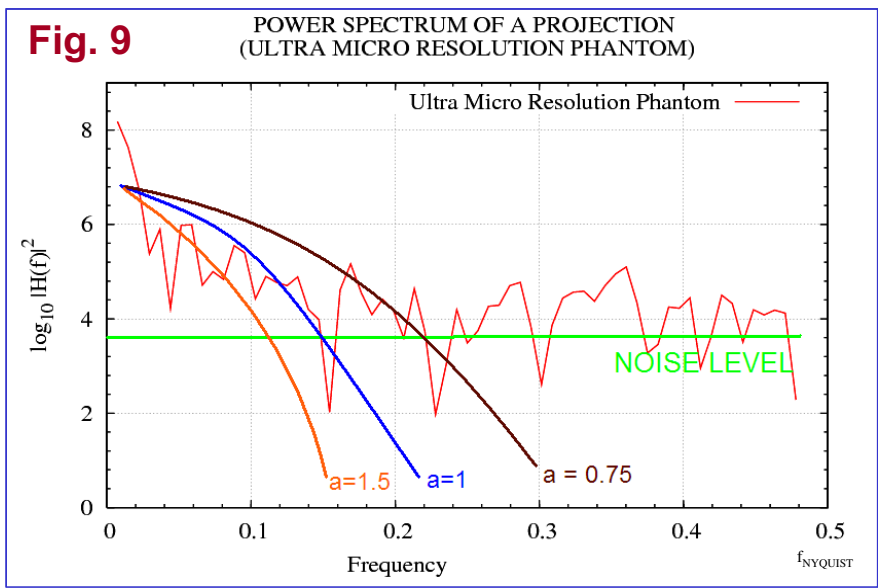

Fig. 9. Power Spectrum of the projected real data and noise level (in green) The different frequency regions that EM ML tries to recover as a function of the FWHM of the TORs are displayed in brown (a 0.75, Thin TORs), blue (a 1, Realistic TOR) and orange (a 1.5, Wide TORs). In this last case, a quasi noise free image is yielded, but with poor resolution.

\section{CONSIDERATIONS FOR THE DESIGN OF PET SCANERS}

Pet Scanners are designed bearing in mind analytical reconstruction methods. For example, high sampling is one of the main design goals. On the other hand, iterative methods are not so sensitive to the sampling employed, because of the width of the TORs. Specific design strategies must be used in order to get optimal results with iterative methods. Reducing the size of the crystals beyond certain point will not further reduce the width of the TORS, while, as the number of counts in a LOR $\mathrm{n}$ is decreased, the relative importance of noise $(\sqrt{ } \mathrm{n} / \mathrm{n})$ will be larger (Fig. 10). This implies that the MSF will not be improved significantly, but the MESF will start to worsen beyond certain minimum crystal section.

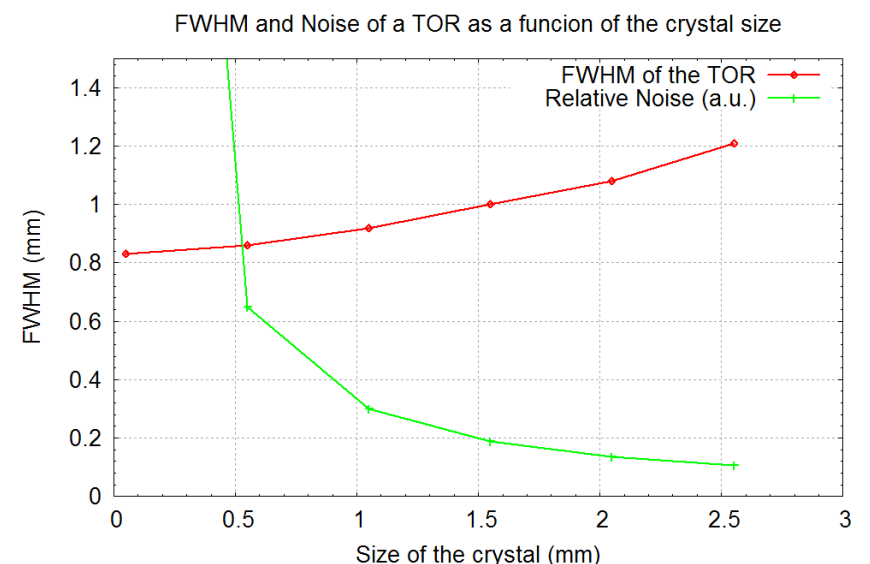

Fig.10. FWHM and Noise of a TOR as a function of the crystal size (in $\mathrm{mm}$ ) It can be noticed that below certain size, it is not worth using smaller crystals, as it will not improve the MESF.

\section{CONCLUSIONS}

The size of the TORs determines, to a much larger extent than the number of LORs or the distance among them, the main properties of small animal PET scanners, like best resolution or the signal to noise ratio achievable.

An analysis of the frequency components found on the projection data and on the reconstructed images provides useful information in order to tune statistical methods.

A quantitative estimate of the effective limiting system frequency is made that is DIFFERENT and more restrictive than the sampling (Nyquist related) frequency limitation. The MSF of any particular system can be estimated a priori, and this estimate can be used to determine the optimal stopping point for the iterative reconstruction method and the maximum achievable resolution from eq. (3).

The most accurate SRM is not necessarily the best choice when iterative reconstruction methods are employed.

\section{REFERENCES}

[1] L. A. Shepp and Y. Vardi, "Maximum likelihood reconstruction for emission tomography,” IEEE Trans. Med. Imag., vol. MI 1, pp. 113 121, 1982.

[2] J. Browne and Alvaro R. De Pierro, "A row action alternative to the EM algorithm for maximizing likelihoods in emission tomography," IEEE Trans. Med. Imag., vol. 15, no. 5, Oct. 1996.

[3] D. L. Snyder, M. I. Miller, L. J. Thomas, Jr., and D. G. Politte, "Noise and edge artifacts in maximum likelihood reconstruction for emission tomography,” IEEE Trans. Med. Imag., vol. MI 6, pp. 228 238, 1987.

[4] D.G. Politte and D. L. Snyder, "The use of constraints to eliminate artifacts in maximum-likelihood image estimation for emission tomography," IEEE Trans. Nuclear Sci., vol. NS-35, pp.608-610, Feb. 1988.

[5] Bettinardi, E. Pagani, M. Gilardi, S. Alenius, K. Thielemans, M. Teras, F. Fazio. "Implementation and evaluation of a 3D one step late reconstruction algorithm for 3D positron emisión tomography brain studies using median root prior," European Journal of Nuclear Medicine, vol. 29, no. 1, pp. 7 18, Jan. 2002.

[6] Eddy T.P. Slijpen and Reek J. Beekman, "Comparison of post filtering and filtering between iterations for SPECT reconstruction," IEEE Trans. Nuc. Sci.,, vol. 46, no. 6, pp. 2233 2238, Dec. 1999.

[7] Glenn D. Boreman "Modulation Transfer Fuction in Optical and Electro Optical Systems" (SPIE Press, 2001).

[8] N. Sabba, G. Di Domenico, E. Moretti, G. Zavattini, A. Del Guerra, Sampling Consideration for high resolution SPECT with the Ferrara YAP (S)PET scanner, IEEE Nuc. Sci. Symposium and Med. Imag. Conference, vol. Conference Record Cd rom, pp. M11 170, Norfolk, Virginia, USA 2002.

[9] J. López Herraiz, S. España, J. J. Vaquero, M. Desco,J. M. Udias, "Full 3D OSEM reconstruction with compressed response of the system", IEEE Nuc. Sci. Symposium and Med. Imag. Conference, vol. Conference Record Cd rom, pp. M05 106, Rome, Italy 2004. 